Material Supplementary

Healthcare resources Unit costs (€)

\section{Medical visits[1]}

Primary care medical visit

Emergency room visit

Specialist medical visit*

Hospitalization (one day)[1]

Supplementary tests[1]

Laboratory tests

Conventional radiology

Diagnostic/therapeutic tests

Pharmaceutical prescription

Indirect costs

Cost per workday lost[2]

\subsection{3}

420.90

$$
22.30
$$

Public Retail Price + VAT

Table S1. Description of unit costs

*Only in pulmonology, cardiology, and internal medicine services

Source of health resources: hospital in-house accounting [1] and INE, Instituto Nacional de Estadística [2]

VAT, value added tax 


\section{References}

1. Sicras-Mainar A, Capel M, Navarro-Artieda R, Nuevo J, Orellana M, Resler G. Real-life retrospective observational study to determine the prevalence and economic burden of severe asthma in Spain. J Med Econ 2020; 23: 492-500.

2. Instituto Nacional de Estadística. Encuesta anual de coste laboral. EACL. Año 2018. https://www.ine.es/prensa/eacl_2018.pdf. Accessed February 8, 2021. 
Table S2. Total costs (EUR) according to overuse and recommended use of SABA and recommended use and underuse of ICSwith or without exacerbations by study groups. Values expressed as mean (SD, standard deviation).

\begin{tabular}{|c|c|c|c|c|c|c|}
\hline \multirow[b]{2}{*}{ Classification } & \multicolumn{2}{|c|}{ SABA } & \multicolumn{2}{|c|}{ ICS } & \multicolumn{2}{|c|}{ Exacerbations } \\
\hline & Recommended use & Overuse & Recommended use & Under use & Without exacerbations & With exacerbations \\
\hline GINA - 1 & $€ 1,204(2,130)$ & $€ 4,708(3,169)$ & --- & & $€ 638(1,620)$ & $€ 4,294(2,954)$ \\
\hline GINA - 2 & $€ 1,689(2,560)$ & $€ 5,248(3,587)$ & $€ 1,706(2,590)$ & $€ 3,108(3,725)$ & $€ 1,015(2,001)$ & $€ 4,784(3,283)$ \\
\hline GINA - 3 & $€ 1,916(2,448)$ & $€ 5,381(3,345)$ & $€ 2,608(3,084)$ & $€ 4,255(2,885)$ & $€ 1,139(1,919)$ & $€ 4,901(2,014)$ \\
\hline GINA - 4 & $€ 2,304(2,589)$ & $€ 6,200(3,406)$ & $€ 3,294(3,395)$ & $€ 5,102(3,541)$ & $€ 1,487(1,907)$ & $€ 5,765(3,273)$ \\
\hline GINA - 5 & $€ 2,849(2,625)$ & $€ 6,973(4,150)$ & $€ 4,617(3,788)$ & $€ 6,024(4,808)$ & $€ 2,150(1,861)$ & $€ 6,676(4,059)$ \\
\hline Total & $€ 1,916(2,495)$ & $€ 5,702(3,542)$ & $€ 2,903(3,255)$ & $€ 4,116(3,446)$ & $€ 1,177(1,911)$ & $€ 5,233(3,295)$ \\
\hline
\end{tabular}

EUR, Euro; GINA, global Initiative for Asthma; ICS,inhaled corticosteroids; SABA, short acting beta-2 agonists. 